\title{
Analysis of soil erosion characteristics in small watersheds with particle swarm optimization, support vector machine, and artificial neuronal networks
}

\author{
Li Yunkai · Tian Yingjie - Ouyang Zhiyun · \\ Wang Lingyan $\cdot$ Xu Tingwu $\cdot$ Yang Peiling $\cdot$ \\ Zhao Huanxun
}

Received: 7 November 2008/ Accepted: 29 August 2009/Published online: 17 September 2009

(C) Springer-Verlag 2009

\begin{abstract}
Sand production by soil erosion in small watershed is a complex physical process. There are few physical models suitable to describe the characteristics of the intense erosion in domestic loess plateau. Introducing support vector machine (SVM) oriented to small sample data and possessing good extension property can be an effective approach to predict soil erosion because SVM has been applied in hydrological prediction to some extent. But there are no effective methods to select the rational parameters for SVM, which seriously limited the practical application of SVM. This paper explored the application of intelligence-based particle swarm optimization (PSO) algorithm in automatic selection of parameters for SVM,
\end{abstract}

Li Yunkai, Tian Yingjie, Ouyang Zhiyun and Wang Lingyan are equally contributed to this paper.

L. Yunkai $(\bowtie) \cdot$ W. Lingyan $\cdot$ Y. Peiling

College of Water Conservancy and Civil Engineering, China

Agricultural University, 100083 Beijing,

People's Republic of China

e-mail: yunkai@cau.edu.cn

W. Lingyan

e-mail: wlyanzi@163.com

Y. Peiling

e-mail: yangpeiling@126.com

L. Yunkai · O. Zhiyun ( $\square)$

State Key Laboratory of Urban and Regional Ecology, Research

Center for Eco-Environmental Sciences, Chinese Academy of

Sciences, 100085 Beijing, People's Republic of China

e-mail: zyouyang@rcees.ac.cn

T. Yingjie

Research Center on Fictitious Economy and Data Science,

Chinese Academy of Sciences, 100080 Beijing,

People's Republic of China

e-mail: tianyingjie1213@163.com and proposed a prediction model by linking PSO and SVM for small sample data analysis. This method utilized the high efficiency optimization property and swarm paralleling property of PSO algorithm and the relatively strong learning and extending capacity of SVM. For an example of Huangfuchuan small watershed, its intensive fragmentation and intense erosion earn itself the name of "worst erosion in the world". Using four characteristics selection algorithms of correlation feature selection, the primary affecting factors for soil erosion in this small watershed were determined to be the channel density, ravine area, sand rock proportion, and the total vegetation coverage. Based on the proposed PSO-SVM algorithm, the soil
$\mathrm{X}$. Tingwu

International College at Beijing, China Agricultural University, 100083 Beijing, People's Republic of China

e-mail: xutingwu2005@yahoo.com.cn

\section{Z. Huanxun}

Institute of Water Conservancy Science of Inner Mongolia, 010020 Hohhot, People's Republic of China

e-mail: zhhuanxun@126.com 
erosion modulus in the small watershed was predicted. The accuracy of the simulation and prediction was good, and the average error was $3.85 \%$. The SVM predicting model was based on the monitoring data of sand production. The construction of the SVM erosion modulus prediction model for the small watershed comprehensively reflected the complex mechanism of soil erosion and sand production. It had certain advantage and relatively high practical value in small sample prediction in the discipline of soil erosion.

Keywords Soil erosion - Particle swarm algorithm . Support vector machine $\cdot$ Small watershed .

Characteristics extraction

\section{Introduction}

Soil erosion is one of eco-environmental issues that have been paid much attention. The prediction of soil erosion is essential for effectively monitoring soil and water loss and assessing water conservation benefit. Models of soil erosion serve as tools for monitoring and predicting soil loss. At present, models of soil erosion have become the research frontiers in soil erosion discipline and effective approaches for quantitative research in soil erosion process. In the last 30 years, models of soil erosion have been consecutively developing by resources integration in various countries, such as Universal Soil Loss Equation (USLE, Wischmeier and Smith 1978), Revised Universal Soil Loss Equation (RUSLE, Renard et al. 1997), Water Erosion Prediction Project (WEPP, Baffalt et al. 1996; Flanagan 2001), LImburg Soil Erosion Model (LISEM, De Roo 1996) and European soil erosion model (EROSEM, Morgan et al. 1998), etc. Scholars in China have fruitful achievements in various research interfaces of soil erosion models (Yin and Chen 1989; Fan 1985; Sun et al. 1988; Jiang et al. 1996; Jin et al. 1991; Tan et al. 1990; Cai et al. 1998; Liu et al. 2002). Tang and Cai (2002), Cai and Liu (2003), Wang et al. (2004) and Ni et al. (2005) conducted systematic induction and summarization on sand production models of soil erosion in China. Generally speaking, in soil erosion research for small watersheds, the majority of the models focused on localization research of various factors in statistical models while few models focused on process-based research. The existence of fewer process-based models suitable for specific situation in China was caused by the extremely complex physical process of sand production in soil erosion and the difficulty in describing the physical mechanism of soil erosion by mathematical approaches.

At the same time, as the soil erosion mechanism was not really clear and the issue of basic data collection was difficult, the physical model suitable for domestic Loess Plateau intense erosion features was extremely few. The soil erosion in small watershed was influenced and restricted by some factors, such as the terrain, soil, runoff, land utilization and so on. In fact, the choice of major effective factors can be regarded as a typical high-dimensional data feature extraction issue. And the issue of predicting the sediment yield of erosion can be regarded as a complex nonlinear regression problem between sediment yield of soil erosion and factors of influence. $\mathrm{Li}$ et al. (2007), using of the USLE equation and China soil erosion prognostic equation, introduced exploringly the support vector machine (SVM) method to construct the small watershed soil erosion modulus prediction model and more satisfactory analog results were obtained. However, the selection of primary soil erosion affecting factors and the rational usage of parameters for SVM still lacked the theoretic basis.

As the difference of treatment problem, the SVM can be named support vector classification (SVC) and support vector regression (SVR). The support vector dealing with regression problems is known as SVR (Deng and Tian 2004). On the basis of extracting soil erosion characteristics from small watershed with intense erosion, this paper exploringly applied the swarm intelligence particle algorithm 'particle swarm optimization' (PSO) for the automatic selection of the SVR parameter, and constructed the PSO-SVR coupling predicting model suitable for small watershed soil erosion modulus prediction.

\section{PSO $-\varepsilon$ SVR Algorithm and realization}

Standard algorithm of $\varepsilon$-SVR

Suppose the regression training sample set is given:

$T=\left\{\left(x_{1}, y_{1}\right), \ldots,\left(x_{k}, y_{k}\right)\right\} \in(X \times Y)^{k}$,

where $\quad x_{i} \in X=R^{n}, y_{i} \in Y=R, i=1,2 \ldots, k$. Select $\varepsilon$-non-sensitive loss function

$c(x, y, f(x))=|y-f(x)|_{\varepsilon}$,

$|y-f(x)|_{\varepsilon}=\max \{0,|y-f(x)|-\varepsilon\}$

Then the corresponding primal optimization problem of SVR is

$$
\begin{array}{ll}
\min _{w \in R^{n}, \xi^{*}, b \in R} \quad & \frac{1}{2}\|w\|^{2}+C \sum_{i=1}^{k}\left(\xi_{i}+\xi_{i}^{*}\right) \\
\text { s.t. } \quad & \left(\left(w \cdot x_{i}\right)+b\right)-y_{i} \leq \varepsilon+\xi_{i}, \quad i=1,2, \ldots, k, \\
& y_{i}-\left(\left(w \cdot x_{i}\right)+b\right) \leq \varepsilon+\xi_{i}^{*}, \quad i=1,2, \ldots, k, \\
& \xi_{i}^{(*)} \geq 0, \quad i=1,2, \ldots, k,
\end{array}
$$

where $(*)$ denotes the simple notation of with $*$ and without $*$. For instance, $\xi_{i}^{(*)} \geq 0$ denotes $\xi_{i} \geq 0$ and $\xi_{i}^{*} \geq 0$, while 
$\xi^{(*)}$ denotes the vector of $\left(\xi_{1}, \xi_{1}^{*}, \ldots, \xi_{k}, \xi_{k}^{*}\right)^{\mathrm{T}}$. Introducing the dual problem for problem (4):

$$
\begin{aligned}
& \min _{\alpha^{(*)} \in R^{2 k}} \quad \frac{1}{2} \sum_{i, j=1}^{k}\left(\alpha_{i}^{*}-\alpha_{i}\right)\left(\alpha_{j}^{*}-\alpha_{j}\right) K\left(x_{i}, x_{j}\right) \\
& +\varepsilon \sum_{i=1}^{k}\left(\alpha_{i}^{*}+\alpha_{i}\right)-\sum_{i=1}^{k} y_{i}\left(\alpha_{i}^{*}-\alpha_{i}\right) \\
& \text { s.t. } \quad \sum_{i=1}^{k}\left(\alpha_{i}-\alpha_{i}^{*}\right)=0 \\
& \quad 0 \leq \alpha_{i}, \alpha_{i}^{*} \leq C, \quad i=1,2, \ldots, k
\end{aligned}
$$

where $K\left(x_{i}, x_{j}\right)$ is the kernel function and the final regression decision function can be obtained by solving this dual problem. In this paper, the RBF kernel function is selected as

$K\left(x_{i}, K_{j}\right)=\exp \left(-\frac{\left|x_{i}-x_{j}\right|^{2}}{2 \sigma^{2}}\right)$

The concrete procedure for this algorithm is as follows:

(1) Suppose the given training set is $T=\left\{\left(x_{1}, y_{1}\right), \ldots\right.$, $\left.\left(x_{k}, y_{k}\right)\right\} \in(X \times Y)^{k}$, where $x_{i} \in X=R^{n}, y_{i} \in Y=$ $R, \quad i=1,2, \ldots, k$

(2) Choose appropriate parameters $\varepsilon, C$ and $\sigma$;

(3) Construct and solve problem (5), and get the solution $\bar{\alpha}=\left(\bar{\alpha}_{1}, \bar{\alpha}_{1}^{*}, \ldots, \bar{\alpha}_{k}, \bar{\alpha}_{k}^{*}\right)^{T} ;$

(4) Construct the decision function:

$f(x)=\sum_{i=1}^{k}\left(\bar{\alpha}_{i}^{*}-\bar{\alpha}_{i},\right) K\left(x_{i}, x\right)+\bar{b}$,

where $\bar{b}$ is calculated by Eqs. (8)-(9): Select $\bar{\alpha}_{j}$ and $\alpha_{k}^{*}$ in $\left(0, \frac{C}{k}\right)$. If the selected value is $\bar{\alpha}_{j}$, then

$\bar{b}=y_{i}-\sum_{i=1}^{k}\left(\bar{\alpha}_{i}^{*}-\bar{\alpha}_{i}\right)\left(x_{i} \cdot x_{j}\right)+\varepsilon$

else

$\bar{b}=y_{k}-\sum_{i=1}^{k}\left(\bar{\alpha}_{i}^{*}-\bar{\alpha}_{i}\right)\left(x_{i} \cdot x_{k}\right)-\varepsilon$

$\varepsilon$-SVR Algorithm of linear programming pattern

Replace $\|w\|_{2}$ in $\varepsilon$-SVR with $\|w\|_{1}$ and get the primal problem:

$$
\begin{array}{ll}
\min _{w, b} & \frac{1}{2}\|w\|_{1}+C \sum_{i=1}^{k}\left(\xi_{i}+\xi_{i}^{*}\right) \\
\text { s.t. } & \left(w \cdot x_{i}\right)+b-y_{i} \leq \varepsilon^{*}+\xi_{i}, \quad i=1,2, \ldots, k, \\
& y_{i}-\left(w \cdot x_{i}\right)-b \leq \varepsilon+\xi_{i}^{*}, \quad i=1,2, \ldots, k, \\
& \zeta_{i}, \xi_{i}^{*} \geq 0, \quad i=1,2, \ldots, k,
\end{array}
$$

Because the sparse solution for $\|w\|_{1}$ exists for the minimization problem, those $[x]_{i}$ with small values of $\left|[w]_{i}\right|$ are treated as unimportant properties. By introducing $w=p-q, \quad p \geq 0, \quad q \geq 0, \quad$ the $\varepsilon$-SVR of linear programming pattern is as follows:

$$
\begin{array}{ll}
\min _{p, q, b, \xi^{(*)}} & \sum_{i=1}^{k}\left(p_{i}+q_{i}\right)+C \sum_{i=1}^{k}\left(\xi_{i}+\xi_{i}^{*}\right) \\
\text { s.t. } \quad & \sum_{j=1}^{k}\left(p_{i}-q_{i}\right)\left(x_{j} \cdot x_{i}\right)+b-y_{i} \leq \varepsilon+\xi_{i}, \quad i=1,2, \ldots, k, \\
& y_{i}-\sum_{j=1}^{k}\left(p_{i}-q_{i}\right)\left(x_{j} \cdot x_{i}\right)-b \leq \varepsilon+\xi_{i}^{*}, \quad i=1,2, \ldots, k, \\
p_{i}, q_{i} \geq 0, \quad i=1,2, \ldots, k, \\
\zeta_{i}, \xi_{i}^{*} \geq 0, \quad i=1,2, \ldots, k,
\end{array}
$$

PSO $-\varepsilon S V R$ Algorithm and realization

PSO was a bionic optimization algorithm proposed by Kennedy (an American social psychologist) and Eberhart (an electrical engineer) in 1995. At the beginning of the algorithm, PSO made particle swarm be initialized as random particles. In each iteration, the particle tailed two extrema to update itself: the first was the best solution which the particle itself found, called the partial extreme point (using pbest to express its position), the other extreme point was the best solution which the entire population found at present, called the overall situation extreme point (using gbest to express its position). After finding these two best solutions, according to formula (12) and formula (13), the particle renewed its owned speed and position. The information of particle $i$ may use d-dimensional representation, the position expression is $Y_{i}=\left(y_{i 1}, y_{i 2}, \ldots, y_{i d}\right)^{T}$, the speed is $V=\left(v_{i 1}, v_{i 2}, \ldots, v_{i d}\right)^{T}$, other vectors are similar, then,

$$
\begin{aligned}
v_{i d}^{k+1}= & w \times v_{i d}^{k}+c_{1} \times \operatorname{rand}_{1}^{k} \times\left(\operatorname{pbest}_{i d}^{k}-x_{i d}^{k}\right)+c_{2} \\
& \times \operatorname{rand}_{2}^{k} \times\left(\operatorname{gbest}_{i d}^{k}-x_{i d}^{k}\right) \\
y_{i d}^{k+1}= & y_{i d}^{k}+v_{i d}^{k+1}
\end{aligned}
$$

In the formula, $v_{i d}^{k}$ is particle $i$ in the $k$ th iteration of the d-dimensional speed; $c_{1}, c_{2}$ are accelerating coefficients, letting $c_{1}=c_{2}=2$; $\operatorname{rand}_{1,2}$ is the random number between 0 and $1 ; x_{i d}^{k}$ is particle $i$ in the $k$ th iteration of the $\mathrm{d}$-dimension of the current location; pbest $_{d}$ is the individual extreme point position where particle $i$ is located in the $\mathrm{d}$-dimensional; gbest $_{d}$ is the overall situation extreme point position where the entire particle group is located in the d-dimensional.

$W$ is the initial weight value of inertia, generally ranging from 0 to 1.4. $w$ can make the necessary reduction of the number of iterative smaller, and $w$ linearly decreases over time.

In order to prevent the particle from being far away from the search space, each dimensional speed of the particle 
can be clamped in $\left[-v_{d \max },+v_{d \max }\right]$. If $v_{d \max }$ is too big, the particle will fly away from the best solution; if too small, it will fall into partially most superior.

The steps of PSO algorithm are as follows:

STEP1. Determine the particle size and particle dimension, and initialize a group of particles, including random speed and random locations.

STEP2. Update the speed and position of each particle according to formula (12) and formula (13).

STEP3. Evaluate the fitness of each particle according to fitness function (by specific optimization problem definition).

STEP4. For each particle, compare its fitness with best position pbest of its own history; take it as the current best position pbest if it is better.

STEP5. For each particle, compare its fitness with its best position experienced in the overall situation; take it as the overall situation best position gbest if it is better.

STEP6. If it satisfies one of the withdrawal conditions, it will withdraw the procedure and return to the current most superior individual and the sufficiency; otherwise it will return to STEP2.

There are three withdrawal conditions: (1) The running number of times is bigger than or equal to the biggest iterative number of times; (2) the running time is longer than or equal to the longest running time; (3) the fitness value is smaller than or equal to the requirement threshold.

$\varepsilon$-SVR has three parameters to be optimized (correspondingly, each particle's dimension is also three dimensional), namely, the penalty constant $C$, non-sensitive parameter $\varepsilon$ and nuclear function parameter $\sigma$. Penalty constant $C$ determines the penalty degree to the sample beyond the requirement of error. The bigger $C$ is the higher fitting accuracy required, which causes the training being more difficult and more time is consumed. The non-sensitive parameter $\varepsilon$ reflects the sensitivity degree of the regression model to the noise contained in the input parameters. The larger $\varepsilon$ is, the lower of the accuracy of model simulation is. The parameter $\sigma$ in the RBF nuclear function represents the standard deviation in Gaussian function, i.e., the width of the function along the direction of the independent variable. Small value of $\sigma$ indicates the good fitting performance of the nuclear function and poor pan-ability variation. According to relevant literature (Xiong and $\mathrm{Xu}$ 2006), the optimization intervals for three parameters are $C \in[0.001,200], \varepsilon \in[0,0.8]$, and $\sigma \in[0.001,5]$.

For realization of PSO $-\varepsilon S V R$, it is equivalent to make the SVR model's structure and the predicting algorithm be inserted into the step of PSO computing adaptation value. The concrete algorithm flow is as follows:
STEP1. If a particle of PSO algorithm module finds to a group of $C, \varepsilon, \sigma$, it will transfer to STEP2.

STEP2. Construct SVR by the group of parameters, carry on the data ten pays proportionately the fork confirmation, and take the standard value as sufficiency value returning the PSO arithmetic module, then transfer to STEP3.

STEP3. If all particles of the PSO algorithm have carried on the STEP2 computation of sufficiency value, then transfer to STEP4, otherwise transfer to STEP1.

STEP4. Renew positional information, the speed information as well as the local extremum of each particle of the PSO algorithm, then transfer to STEP5.

STEP5. The PSO algorithm module judges whether reaching the withdrawal condition or not. If reaching the withdrawal condition, it will withdraw and return the optimal-adaptive value, otherwise transfer to STEP1.

\section{PSO - ESVR Prediction model of soil erosion modulus in small watershed}

The basic situation of Huangfuchuan

Huangfuchuan was a sandy tributary in middle reaches of the Yellow River with watershed area of $3,240 \mathrm{~km}^{2}$, and an annual average sand supply of 50 million tons to the Yellow River. Huangfuchuan small basin originated in southern Inner Mongolia, and it was located in the southeastern part of the Erdos Plateau, the northern edge of the Loess Plateau (Fig. 1). The average precipitation in Huangfuchuan small basin was $389.5 \mathrm{~mm} /$ year as the maximum rainfall in July and the minimum rainfall in November. The greatest precipitation was in the fourth consecutive month in the flood season, whose precipitation accounted for $78 \%$ of annual precipitation, and over about $50-60 \%$ of the rainfall concentrated in July and August. Rainstorm of Huangfuchuan had the most obvious characteristics of short, over size, strength, and inter-annual variation. The maximum rainstorm water was $24.3-76.7 \%$ of all the flood season, and the sediment yield rainstorm flood sediment was 37.6-95.3\% (Xu et al. 2000).

The whole basin has 500 small basins, the area of which are between $1 \mathrm{~km}^{2}$ and $50 \mathrm{~km}^{2}$. The soil erosion situation of so fragile land and so strong erosion was regarded as the "worst situation of the globe" (Jin et al. 1991; Zhao 1998), with the ravine density of $6.7 \mathrm{~km} / \mathrm{km}^{2}$, channel frequency of $34 / \mathrm{km}^{2}$, ravine splitting degree of $35.9 \%$. Considering the differences in surface material 
Fig. 1 Study area

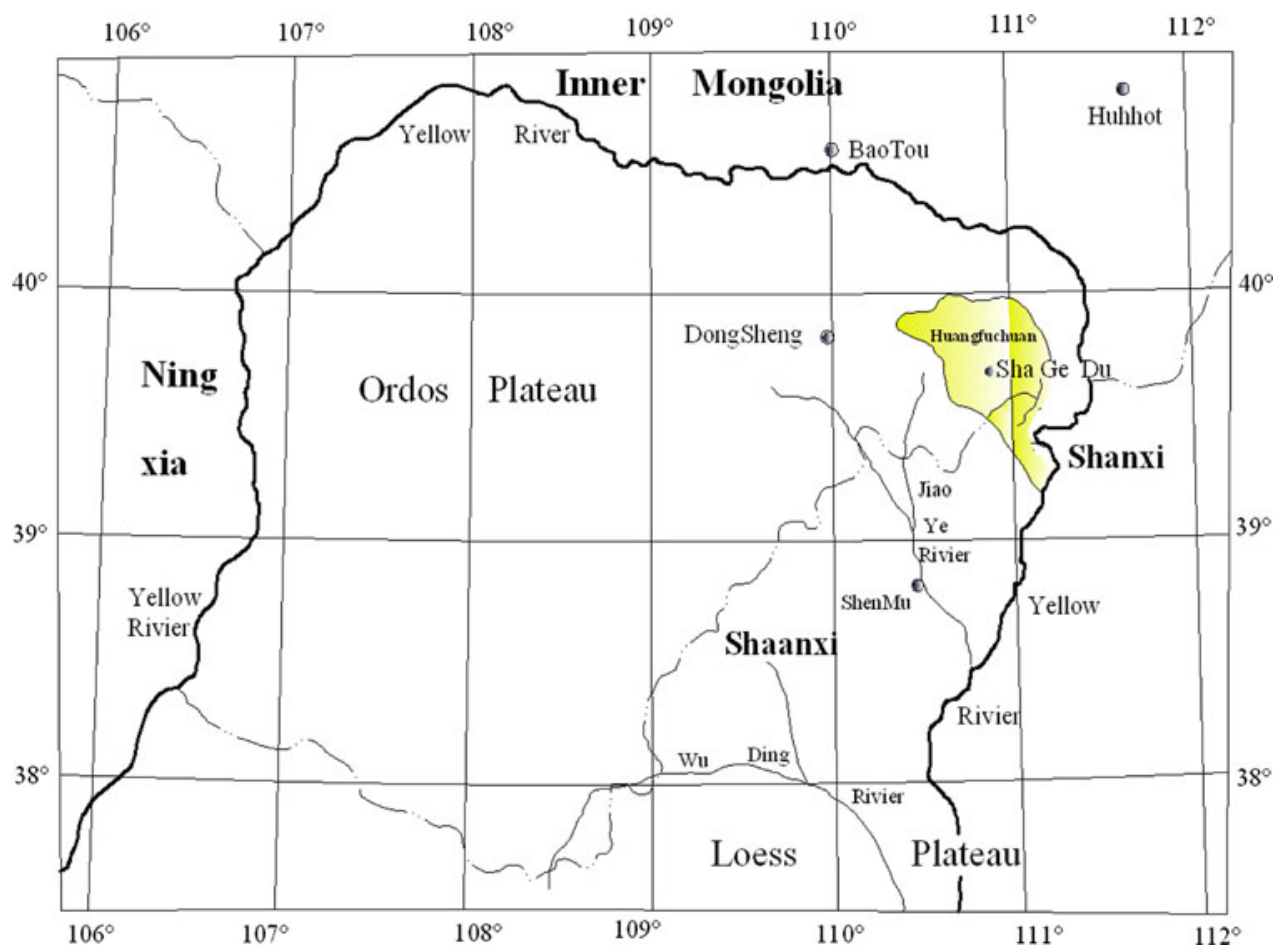

and erosion intensity, they were divided into three types (Fig. 2). The first type was the fierce water erosion small basin, with the sand rock in the trench appeared basically and in the slope exposed in the laminated shape, and the thin layer loess was distributed with laminated shape. Its erosion modulus generally surpassed $20,000 \mathrm{t} / \mathrm{km}^{2}$ a (Fig. 2a). The second type was the intense water erosion small basin, with the sand rock in the trench appeared and the slopes were covered with the loess and the piece sand. Its erosion modulus was 10,000-20,000 t/km a (Fig. 2b). The third type was the mixed water and wind erosion small basin, with a large distribution of sandy land and piece sand. Its erosion modulus was generally smaller than $10,000 \mathrm{t} / \mathrm{km}^{2}$ a (Fig. 2c).

The huge soil erosion treatment campaign was ongoing at levels of state, local authority and individuals. Because of lack of quantitative research on soil erosion in small watersheds, a uniform soil erosion modulus of $18,000 \mathrm{t} / \mathrm{km}^{2}$ a was used in soil erosion treatment planning, engineering design and benefit evaluation in the comprehensive soil erosion treatment campaign. Actually, the difference in erosion factors could cause the erosion modulus to be several folds to even tens of folds different (Tan et al. 1990). The existence of the significant error in estimating soil erosion modulus made it difficult to accurately assess the benefit of small watershed treatment campaign. Hence, the establishment of soil erosion prediction model for small basins played an important role in the ecological construction and comprehensive treatment in Huangfuchuan watershed.
Feature extraction of soil anti-erosion in small watershed

\section{Soil erosion modulus in small watershed}

The investigated sediment was not filled up at the same time, so the measured multi-annual mean erosion modulus $Y_{1}$ of each small watershed was different from $R$, leading to $Y_{1}$ including the quantity difference caused by $R$. In order to eliminate the influence of $R$ on $Y_{1}$ and caused $Y_{1}$ to correspond to each multi-annual mean value $R$, the $Y_{1}$ must be revised with reference to the local rainfall computation $\mathrm{R}$ and their relative relation. Based on the linear correlation between $Y$ and $R$, the equation below obtains multi-annual mean erosion modulus corrected value $Y_{2}$ correspondingly multi-annual mean $R$ value:

$Y_{2}=\frac{Y_{1}}{R_{1}} \cdot \bar{R}$

$R_{1}=\sum E I_{30}$

where $R_{1}$ is the annual mean $R$ value correspondingly $Y_{1}$, $\mathrm{MJ} \mathrm{mm} / \mathrm{hm}^{2} / \mathrm{h} / \mathrm{a} ; E$ is the total energy of one rainfall to the land, $\mathrm{MJ} \mathrm{hm}{ }^{2} . I_{30}$ is the biggest rainfall intensity in one rainstorm within $30 \mathrm{~min}$.

\section{Upper factors and its data mining}

The main upper factors affecting soil erosion in Huangfuchuan watershed were as follows: (1) terrain factors: $S_{1}$ 

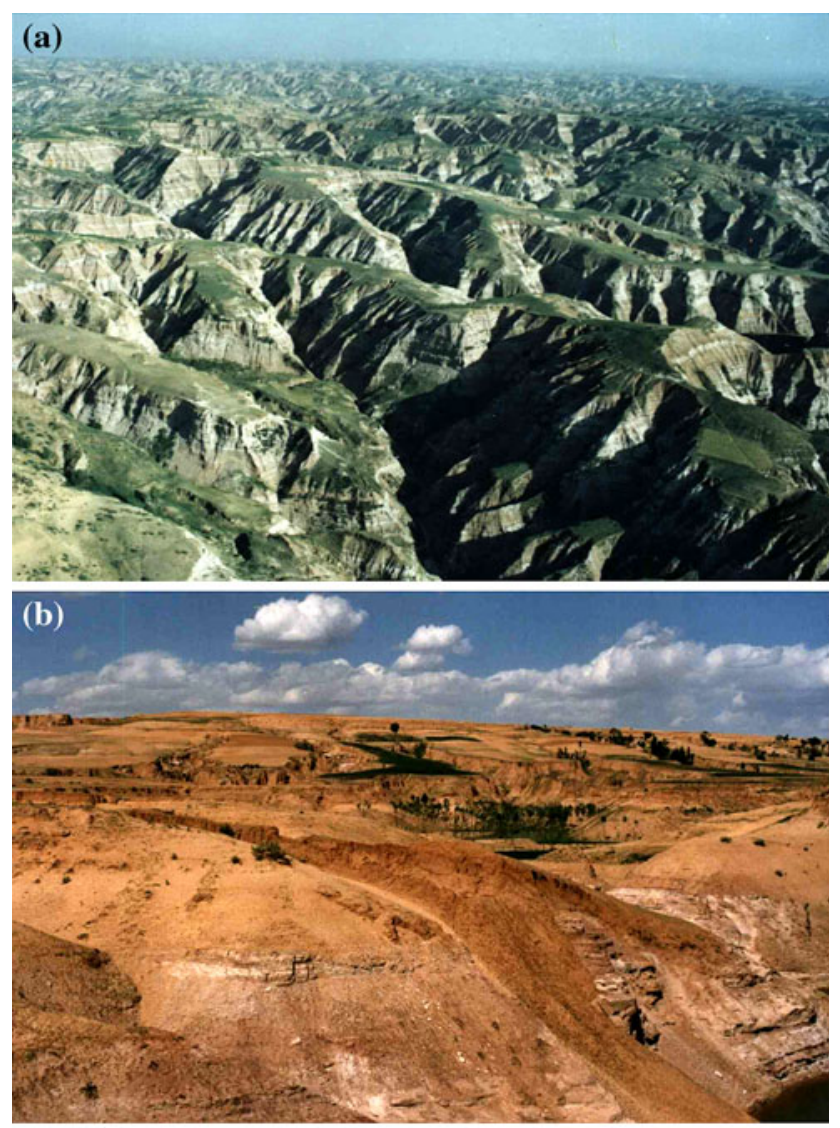

(c)

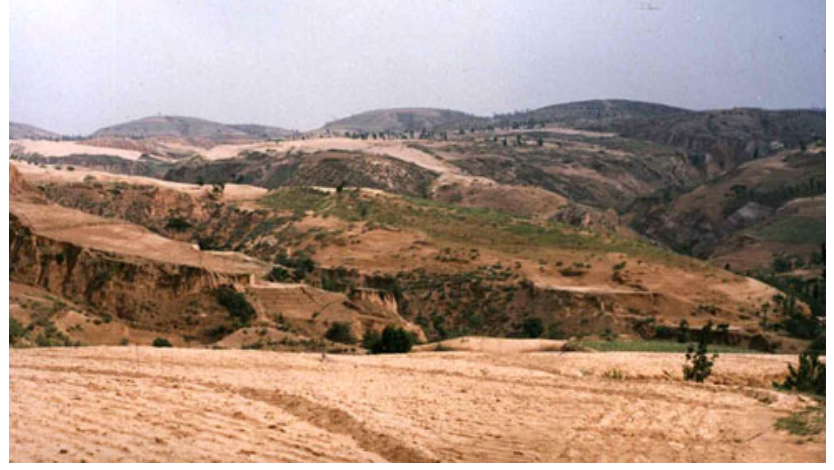

Fig. 2 Soil erosion survey of Huangfuchuan watershed

channel density $/ \mathrm{km}$ per $\mathrm{km}^{2}, S_{2}$ ravine area/ $\%, S_{3}$ average slope ${ }^{\circ}, S_{4}$ land vegetation coverage $/ \%, S_{5}$ topographical ratio/\%; (2) soil factors: $S_{6}$ sand rock proportion/\%, $S_{7}$ sandy soil proportion/\%, $S_{8}$ loess proportion $/ \%, S_{9}$ chestnut soil proportion/\%; (3) vegetation factors: $S_{10}$ total vegetation coverage (\%), $S_{11}$ slope vegetation coverage $/ \%, S_{12}$ ravine vegetation proportion/\%; (4) Other: $S_{13}$ watershed area $/ \mathrm{km}^{2}$, etc. Based on the discussion above, the on-thespot investigation on Huangfuchuan basin was executed to accumulate data of the reservoir and dam of small basin sedimentation. With the platform of Arcview GIS, the data necessary affecting factors were extracted from the colorful infrared aeronautical picture of 1:50,000, colorful infrared aeronautical picture of $1: 10,000$, terrain map of $1: 5000$ and terrain map of 1:50,000. Randomly select various small watersheds and constite 449 samples of 13 inputs and 1 output. In order to avoid the influence of difference among factors, the parameters of input and output were normalized. The indexing treatment was executed to make the values of parameters between 0 and 1 , which was calculated with the difference between the maximum and the minimum for every parameter (Deng and Tian 2004). Then 247 samples were randomly selected from the all as learning sample sets, the rest were used for test sample sets.

\section{Feature extraction algorithm}

Feature selection algorithm was used to choose small number of significant features from the numerous features, retaining the category message as many as possible when reducing the quantity of features. The feature selection algorithm adopted in this paper is correlation feature selection (CFS) (Mark 2000). It attempted to evaluate the degree of correlation between the feature subset and the label through the computation of relevance. After selecting the sandstone percentage as the initial attribute, the feature selection experiment of CFS + BestFirst was carried out. Three kinds of search directions were used including the backward search (CFS + BestFirst + Backward), the forward search (CFS + BestFirst + Forward), and the bidirectional search (CFS + BestFirst + Bi-Direction) to carry out ten of fold inspections. The threshold accounting for the percentage was set to be $100 \%$ in the result of ten of folds. In the above three types of search directions, when the search direction was the Backward, the selected features subset had the smallest dimension; and when the search direction was Forward, the selected feature subset had moderate dimension; when the search direction was Bi-Direction search, the selected feature subset had the largest dimension. By analysis on experimental results to Backward, Forward, and the Bi-Direction, take the ultimate intersection of the three as feature subset.

\section{Results and discussion}

\section{Results}

\section{Feature selection of erosion surface factor} of Huangfuchuan small basin

The threshold accounting for the percentage was set to be $100 \%$ in the result of ten of folds. The intersection of three kinds of research experimental results was taken as the 
final feather subset. By calculation, the final feather subset included four attributes, which were the channel density, ravine area, sand rock proportion and total vegetation coverage. Statistical results of the correlativity between corrosion factors and soil erosion modulus about the four small basins were shown in Fig. 3. Then, SPSS software was applied to carry out the correlation test (Table 1). Table 1 showed the correlativity of thirteen factors with the erosion modulus, and it indicated that the four selected factors had an obvious correlativity with the erosion modulus. The fact proved the accuracy of the feature selection. Density of the ditch net, the cutting of crack, the percentage of sandstone, and the total vegetation cover had positive correlativity, and the correlativity of the percentage of sandstone was the best.

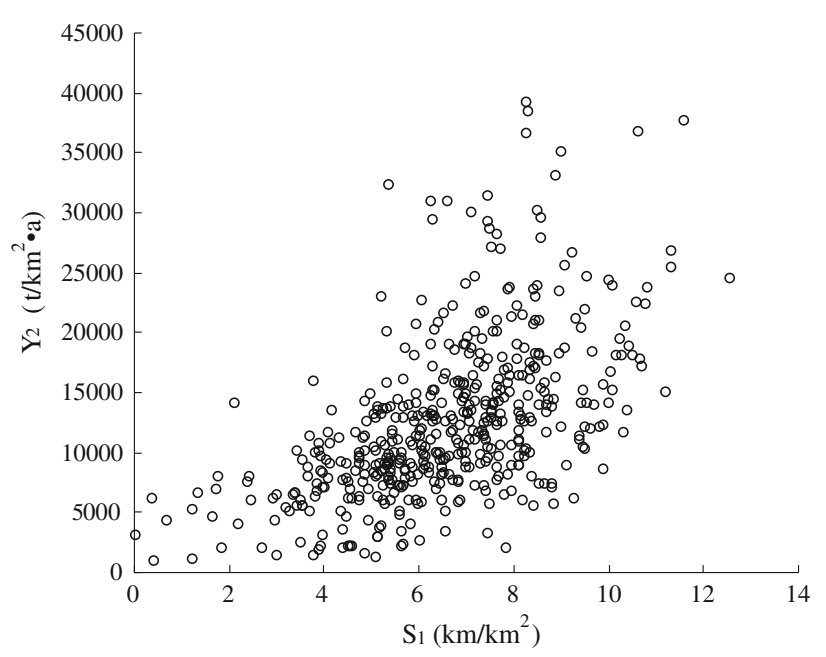

(a)

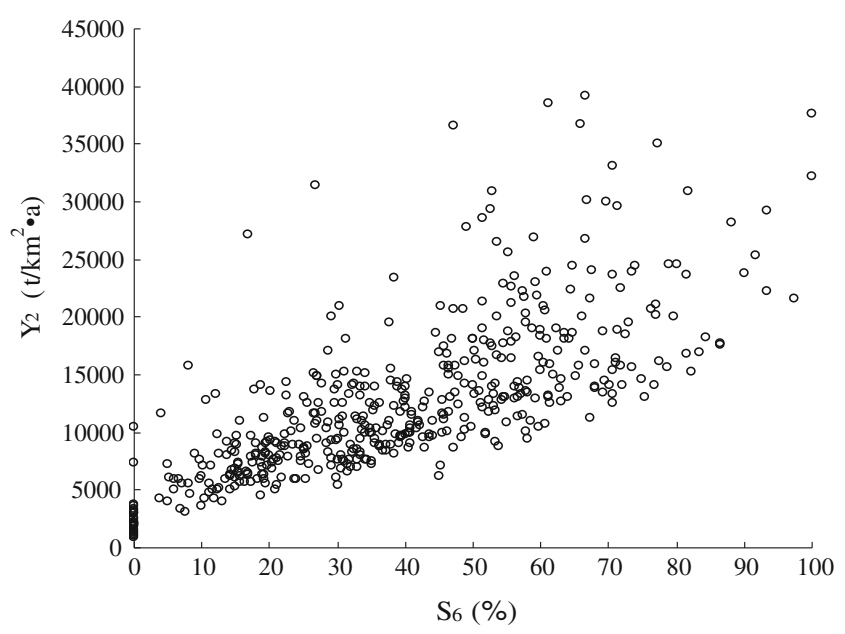

(c)
The simulation of PSO-SVM model predicting soil erosion modulus

The parameters of PSO algorithm were set as follows: the particle dimension d was 3 , the number of population $I$ was 20 , initial inertia weight $W$ was 0.9 , the biggest iterative frequency $K_{\max }$ was 2,000 , and the biggest running time was $\infty$. The value of the terminal sufficiency was equal to 0.0 , the maximum speed of the particle $v_{d \max }$ was 10 , and the biggest position value of the particle $x_{d \max }$ was 100 . Finally, the optimum value of sufficiency was $1,093.8368$, and the most superior parameter $C=150.69, \varepsilon=0.0119$, $\sigma=0.1191$. According to these parameters, the predicting model was obtained by the training, and the predicting results with testing data set and its comparison with the

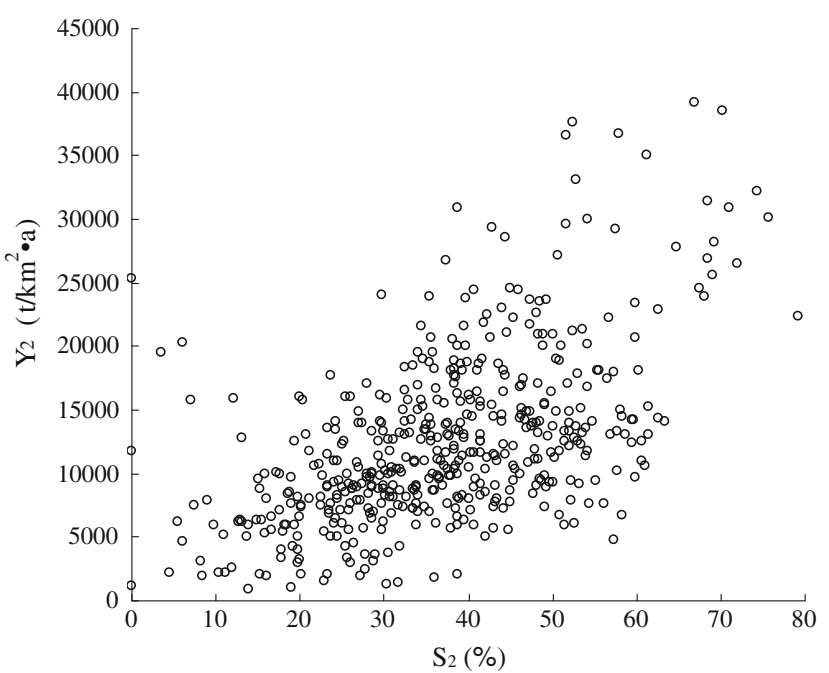

(b)

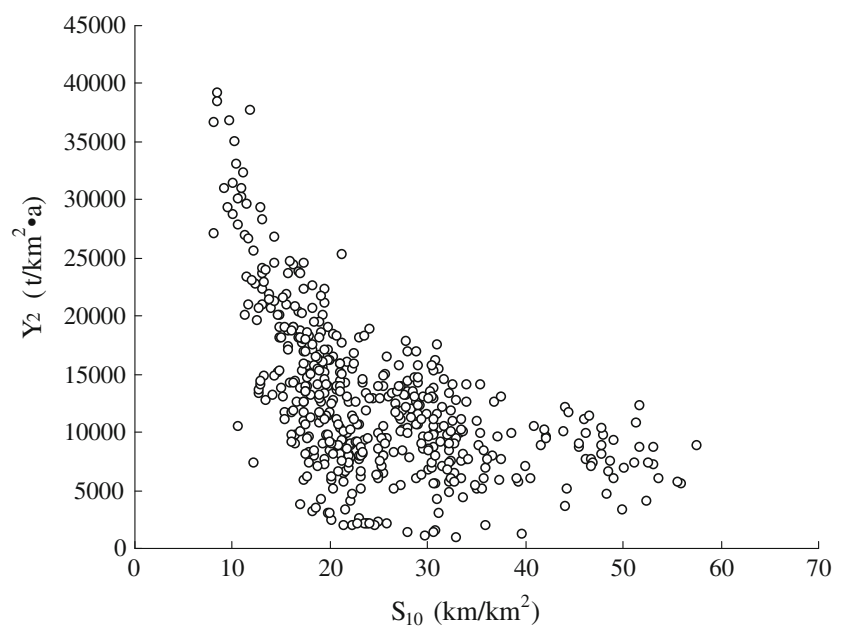

(d)

Fig. 3 Correlativity between upper factors with the modulus of soil erosion. a Channel density. b Ravine area. c Sand rock proportion. d Total vegetation coverage 
Table 1 Pearson test of correlation

\begin{tabular}{llllllll}
\hline Item & $\mathrm{S}_{1}$ & $\mathrm{~S}_{2}$ & $\mathrm{~S}_{3}$ & $\mathrm{~S}_{4}$ & $\mathrm{~S}_{5}$ & $\mathrm{~S}_{6}$ & $\mathrm{~S}_{7}$ \\
\hline Pearson correlation & $0.556^{* *}$ & $0.553^{* *}$ & $0.170^{*}$ & $-6.96 \mathrm{E}-02$ & -0.097 & $0.746^{* *}$ & $-0.282^{* *}$ \\
Sig. $(t$-tailed) & 0.000 & 0.000 & $0.00 \mathrm{E}+00$ & 0.123 & 0.031 & 0.000 & $0.00 \mathrm{E}+00$ \\
Covariance & $7,485.825$ & $62,136.095$ & $16,287.72$ & $-8,203.00$ & $-15,950.00$ & $110,704.694$ & $-52,830.00$ \\
$N$ & 494 & 494 & 494 & 494 & 494 & 494 & 494 \\
\hline Item & $\mathrm{S}_{8}$ & $\mathrm{~S}_{9}$ & $\mathrm{~S}_{10}$ & $\mathrm{~S}_{11}$ & $\mathrm{~S}_{12}$ & $\mathrm{~S}_{13}$ & - \\
\hline Pearson correlation & $-0.303^{* *}$ & $-0.114^{*}$ & $-0.540^{* *}$ & $-0.282^{* *}$ & $-0.237^{* *}$ & $-4.49 \mathrm{E}-02$ & - \\
Sig. $(t$-tailed) & 0.00 & 0.01 & 0.00 & 0.00 & 0.00 & 0.32 & - \\
Covariance & $-50,080.00$ & $-7,156.00$ & $-36,650.00$ & $-1,683.00$ & $-15,140.00$ & $-2,586.00$ & - \\
$N$ & 494 & 494 & 494 & 494 & 494 & 494 & - \\
\hline
\end{tabular}

Note. * Mean values are significantly different at $P_{0.05} ; * *$ mean values are significantly different at $P_{0.01}$

simulated value with PSO-ANN (artificial neural network) or ANN model was shown in Fig. 4. The BP neural network model, which was the most widely used ANN model, was applied in simulating the soil erosion quantity. The error of the training sample set was 0.001 . The learning rate was 0.10 . The momentum factor was 0.70 . The $\max$ learning times was 10,000 . It was realized with the MATLAB software package. By the training with neural network, when the learning times approached to 8,400 , the error of the training sample set arrived at 0.00098 which was lower than the predetermined error. The neural network was to convergence, and the training was stopped. The simulation precision of soil erosion quantity prognostic equation simulated by PSO-SVM was extremely high, which was obviously higher than PSO-BP ANN and BP ANN. The main reason was that the SVM had solved the problem of over learning with the structural risk minimization principles. The fitting linear of results over the origin approached to the straight line of $y=x$, and the average prediction error was $3.85 \%$ in testing data.

\section{Discussions}

Soil erosion was the main reason which caused degeneration and even thorough destruction of the land resource. Soil erosion of the small basin was affected and restricted
Fig. 4 The error analysis between measurement values and predicting values

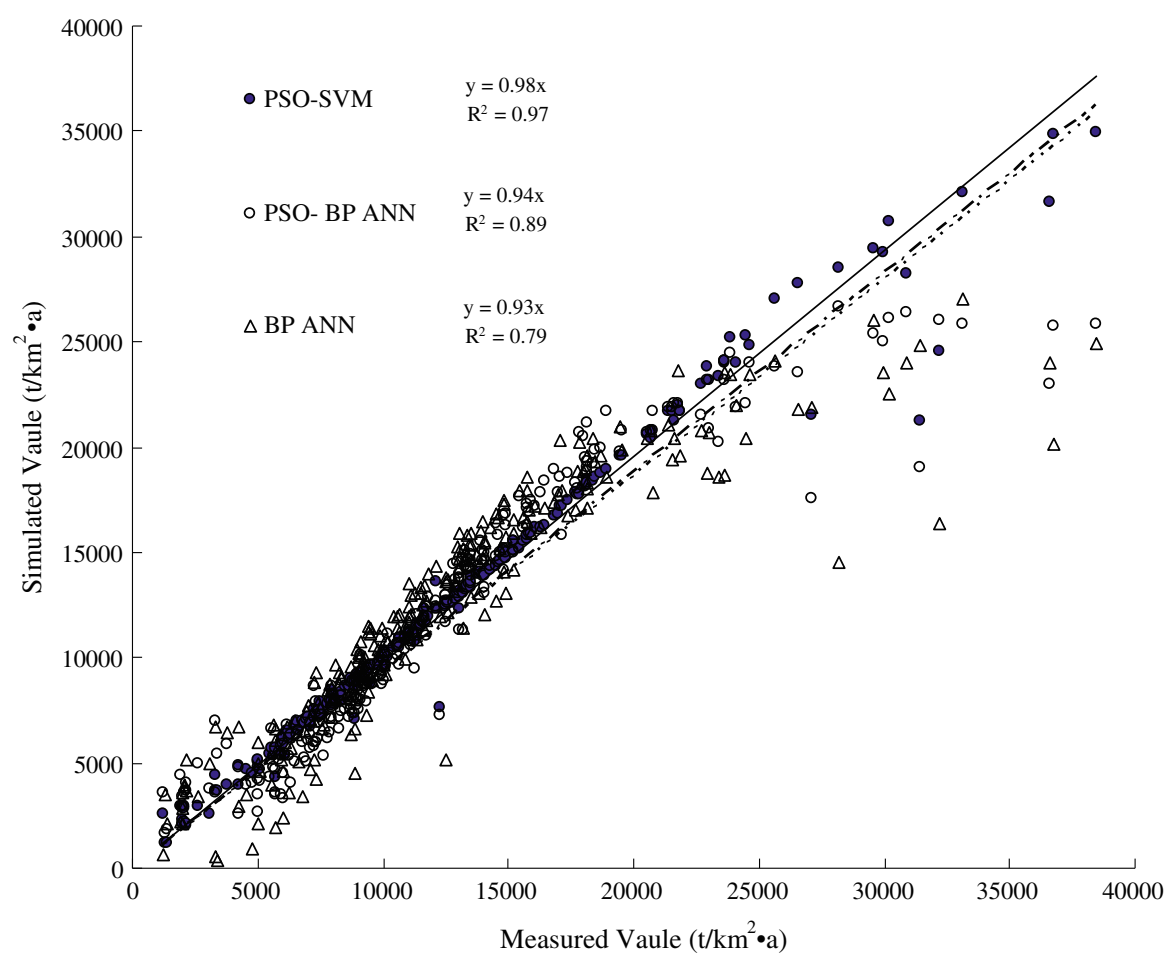


by many kinds of factors, e.g., the terrain, the soil, the runoff, the land utilization, etc. The soil erosion predicting models were focused widely from various countries, as a significant technique tool of understanding the progress and intensity of soil erosion, mastering the quality tendency of land resource, guiding how to use the land resource reasonably, and managing and maintaining the humanity longterm living environment. Over the years, the scholars have conducted a great deal of researches, and a series of empirical statistical models and physical models have been established. The complexity and the applicability of the models were different. Such as empirical statistical models, constructing the soil erosion equations and selecting the main factors mainly depended on the test data and mathematical statistics. So the soil erosion was generally simpler and was always easier to apply. But the application areas of empirical parameters were limited, so as to the accuracy of simulation was difficult to control. While the physical models described the process of soil erosion with the principles of hydrology, hydraulics, soil science and other relevant subjects, they were used to predict the amount of soil erosion in a given period with the rainfall and runoff conditions. The physical models had good generality. But the influencing factors of soil erosion and sediment process in small basin were numerous, and the mechanism was very complex. So it was difficult to describe the progress in a mathematic way. And these models needed a large number of local observed data. Especially in developing countries, the limitation in gathering the needed data made the modeling difficult. The soil erosion in small basin was the comprehensive result of various factors. The issue of sand production prediction was actually the issue of the complex nonlinear regression between sand production and the impacting factors. In recent years, many of the machine learning theories, such as ANN and SVM, have been used successfully to deal with the complex connection of input and output. It was used widely in many areas, and it may become the effective method to predict the soil erosion quantity.

But the problem is how to select typical characteristics from the vast data. This paper exploringly carried out a feature extraction of the soil erosion factor of small basin which used the CFS feature selection algorithm, and made clear which surface factors had affected the soil erosion of Huangfuchuan small basin. The surface factors mainly included the channel density, ravine area, sand rock proportion, and total vegetation coverage. The three factors (channel density, ravine area and total vegetation coverage) reflected the effects of the vegetation and terrain on the soil erosion, which was consistent with previous studies (Jin et al. 1991; Tang and Cai 2002; Cai and Liu 2003; Wang et al. 2004; $\mathrm{Li}$ et al. 2007). The typical characteristics of Huangfuchuan small basin and even the whole loess hilly- gully region were great channel density and ravine area. And the whole small basin presented intensive hilly-gully physiognomy. The channel density and ravine area were selected just for reflecting this characteristic. But generally speaking, the sand rock proportion had a significant impact on erosion among these factors, which was not consistent with previous studies (Jin et al. 1991). The main reason for cutting down the factors was that the local sand rock exposes in steep slopes suffered from the water and gravity eroding. Because the agent of erosion was strong, and the anti-water erosion of sand rock was very weak, the erosion was stronger than in other ground regions. It had become the main source of erosion sediment in small basin. The permeability of sandstorm soil was high and the ability of anti-water erosion was strong, so the relative agent of erosion was weak, and the loess was situated between it. The characteristics of the unique erosion in Huangfuchuan small basin were reflected, and even for the whole loess hilly-gully region and similarity area, which was mainly located in the middle reaches of the Yellow River and the Loess Plateau of northern regions, covering an area of $22.74 \mathrm{~km}^{2}$ (Fu et al. 2002).

There was also an another problem that its simulation precision was affected easily by the selected parameters, which seriously hindered the practical application of SVM and ANN. So it is necessary to select the optimal parameters with optimization algorithms. It can reduce the blindness of trial calculation and improve the simulation precision. The PSO algorithm got more attention with easily arithmetic, global convergence, and rapid calculation. And it can rapidly get the optimal parameters with PSO search. There were a lot of successful reports about the application of PSO-SVM in feature selection (Cheng and Jian 2008), fault diagnostics (Sheng and Fu 2007). But there were only a few reports in Geoinformatics (Wei 2008). This article used the PSO-SVM coupling model to predict soil erosion modulus of the small basin and achieved an excellent result, which was the exploration firstly applied in predicting soil erosion. The simulation average error was $3.85 \%$, and obviously it was higher than the soil erosion modulus prediction in the previous reports (Jin et al. 1991; Li et al. 2007). The main reason for this situation was that SVM based on the structural risk minimization principles and PSO algorithm with global convergence could primely ensure the rationality of parameter identification. As for the prediction of sand production from soil erosion, the SVM model could be established on the basis of annual sand production data. Learning the historical data could help to identify the complex sand production mechanism and realize the computer simulation. It was not an empirical formula but a comprehensive reflection on the complex sand production mechanism. By linking the PSO algorithm and the SVM algorithm, the 
SVM achieved optimum choice of parameters. This prediction model could be used for water and soil conservation and the calculation of the average erosion modulus of multi-year in planning and designing the silt dam project in Huangfuchuan small basin. It could also be used for calculating the benefit of reducing sand in the small basin and drawing up the soil erosion figure of small basin unit, etc. But the prediction model of this article was an empirical equation established by the sediment data of Huangfuchuan small basin, it could be used in the whole loess hilly-gully region and similar areas. And the idea and method could also be referenced by other small basins. Because the model did not not consider thoroughly on the measure of water and soil conservation as well as the complexity and the uncertainty of corrosion which produced the sand, the application of PSO-SVM prediction model demanded further research.

Acknowledgments We are grateful for financial support by the Program for Changiiang Scholars and Innovative Research Team in University (PCSIRT) (No.IRT0657), National Basic Research Program of PR China (973 Project, 2009CB421100), the National Natural, Science Foundation for Innovation Team of China (No. 40701189), "11th Five-year" National Key Project for S\&T of China (2006BAC01A01) and the Science Fund of China Postdoctor (No.20080430072).

\section{References}

Baffalt C, Nearing MA, Nicks AD (1996) Impact of GLIGEN parameters on WEPP predicted average soil loss. Trans ASAE 39(2):1001-1020

Cai QG, Liu JG (2003) Evolution of soil erosion models in China. Prog Geo 22(3):230-242

Cai QG, Wang GP, Chen YZ (1998) Process and simulation of soil erosion in Loess Plateau watershed. Science Press, Beijing, pp 108-110 (in Chinese)

Cheng LH, Jian FD (2008) A distributed PSO-SVM hybrid system with feature selection and parameter optimization. Appl Soft Comput 8:1381-1391

De Roo APJ (1996) The LISEM project: an introduction. Hydrol Process 10:1021-1025

Deng NY, Tian YJ (2004) New method in data mining-SVM. Science Press, Beijing, pp 214-218

Fan RY (1985) Study on the calculating the soil erosion quantity of watershed in the middle of Yellow River. Sci Water Soil Conserv 2:12-18 (in Chinese with English abstract)

Flanagan DC (2001) WEPP CD-ROM

Fu BJ, Chen LD, Qiu Y, Wang J, Meng QH (2002) Land use structure and ecological process in the loess hilly-gully region. Commerce Press, Beijing, p 24

Jiang ZS, Wang ZQ, Liu Z (1996) Quantificational study on the space variety of soil erosion in the Loess hill area. J Soil Eros Soil Water Conserv 2(1):1-9 (in Chinese with English abstract)
Jin ZP, Zhao HX, He T, Zhao HX (1991) An approach to the equation predicting soil erosion of small watershed in Huangfuchuan area. J Soil Water Conserv 5(1):8-18 (in Chinese with English abstract)

Li YK, Yang PL, Tian YJ, Ren SM, Zhao HX (2007) Application of support vector regression method in predicting soil erosion intensity of small watershed in the insensitive erosion areas. J Beijing For Univ 29(3):93-98 (in Chinese with English abstract)

Liu BY, Zhang KL, Xie Y (2002) An empirical soil loss equation/ process of soil erosion and its environment effect, vol II 12th ISCO. Tsinghua Press, Beijing, pp 21-25

Mark AH (2000) Correlation-based feature selection for discrete and numeric class machine learning. In: Proceedings of seventeenth international conference on machine learning. Morgan Kaufmann Publishers Inc., Menlo Park, pp 359-366

Morgan RPC, Quinton JN, Smith RE, Smith RE, Govers G, Poesen JWA, Auerswald K, Chisci G, Torri D, Styczen M E (1998) The European soil erosion model (EUROSEM): a dynamic approach for predicting sediment transport from fields and small catchments. Earth Surf Process Landf 23:527-544

Ni JP, Xie CY, Wei CF, Xie DT (2005) Recent development of predictive model of soil erosion. Sci Water Soil Conserv 3(1):66-71 (in Chinese with English abstract)

Renard KG, Foster GR, Weesies GA, McCool DK, Yoder DC (1997) RUSLE a guide to conservation planning with the revised universal soil loss equation. USDA Agricultural Handbook no. 703

Sheng FY, Fu LC (2007) Fault diagnostics based on particle swarm optimization and support vector machines. Mech Syst Signal Process 21:1787-1798

Sun LD, Sun BP, Chen Y (1988) The predicting equation of soil erosion quantity of Loess hill ravine area watershed in XiJi County. Trans Nat Res 3(2):141-153 (in Chinese with English abstract)

Tan LQ, Chen GX, Cai MY (1990) Mathematical modeling of soil erosion in the Loess hill area. J HEHAI Univ 18(6):10-16 (in Chinese with English abstract)

Tang ZH, Cai QG (2002) Review on the studies of Chinese main soil erosion and sediment yield models. J Mt Sci 20(4):466-475 (in Chinese with English abstract)

Wang ZL, Huang XH, Niu ZH (2004) Review of models of soil erosion and sediment yield in catchments in China. Res Soil Water Conserv 11(4):28-33 (in Chinese with English abstract)

Wei CH (2008) Rainfall forecasting by technological machine learning models. Appl Math Comput 200:41-57

Wischmeier WH, Smith DD (1978) Predicting rain fall erosion losses. USDA Agricultural Handbook no. 537

Xiong WL, Xu BG (2006) Study on optimization of SVR parameters selection based on PSO. J Syst Simul 18(9):2442-2445 (in Chinese with English abstract)

$\mathrm{Xu} \mathrm{JH}, \mathrm{Lv}$ GD, Zhang SL (2000) The region delineation and principle of sand supply in middle reaches of Yellow River. Yellow River Press, Zhengzhou, 215 pp

Yin GK, Chen QL (1989) The characteristic index and statistical pattern of soil erosion in Loess Plateau watershed. Trans Geo 44(1):32-46 (in Chinese with English abstract)

Zhao HX (1998) Reconsideration on the soil erosion laws in HuangFuchuan watershed-application of soil erosion estimation models for small watersheds. Res Soil Water Conserv 5(3):101-105 